\title{
Fe3O4-loaded Ion Exchange Resin for Chromatographic Separation of Boron Isotopes: Experiment and Numerical Simulation
}

\author{
Guo Xianghai ${ }^{1}$, Qingfeng Wang ${ }^{1}$, Tao Chen $^{1}$, Peng Bai ${ }^{1}$, and Jiafei Lyu ${ }^{1}$ \\ ${ }^{1}$ Tianjin University
}

August 31, 2020

\begin{abstract}
Fe3O4-loaded ion exchange resin composites (Fe3O4@Resin) were optimally constructed through ion exchange and co-precipitation of $\mathrm{Fe} 2+$ and $\mathrm{Fe} 3+$ on strong acid ion exchange resin. The as-synthesized Fe3O4@Resin composite was sophisticatedly characterized and investigated for 10B/11B separation including effect of $\mathrm{pH}$, kinetics and isotherms through batch adsorption experiment which can be well described by pseudo-second order kinetics and Langmuir model. In the chromatographic column packed with Fe3O4@Resin, 10B was selectively retained with a high dynamic separation factor of 1.312. Considering the consistency between simulated and experimental breakthrough curves within Fe3O4@Resin packed column, chromatographic 10B/11B separation performance was simulated under various conditions which were further optimized by response surface methodology method. Consequently, the annual yield of 10B reached the maximum of $612 \mathrm{~g}$ with feed concentration of 7.567 $\mathrm{g} \cdot \mathrm{L}-1$, flow rate of $38.57 \mathrm{~mL} * \mathrm{~min}-1$, the length of column of $45 \mathrm{~cm}$. In addition, five-cycle adsorption/regeneration experiments indicated its merit of reusability.
\end{abstract}

\section{Hosted file}

Manuscript.doc available at https://authorea.com/users/354910/articles/478377-fe3o4-1oadedion-exchange-resin-for-chromatographic-separation-of-boron-isotopes-experiment-andnumerical-simulation 